\title{
Contributions to some cavitation problems in turbomachinery
}

\author{
VIJAY H ARAKERI
}

Department of Mechanical Engineering, Indian Institute of Science, Bangalore 560012 , India

e-mail: vijay@mecheng.iisc.ernet.in

MS received 10 February 1999; revised 29 October 1999

\begin{abstract}
In the present article, three problems associated with cavitation in turbomachinery are discussed. The first one deals with the potential application of recent understanding in cavitation inception to similar problems in turbomachinery. The second considers the thermodynamic effects in developed cavitation. This has relevance to turbopump operation using fluids other than water. Old correlations to predict the above effect are summarized and a new correlation is proposed. Lastly, the possible methodology to predict pump cavitation noise is outlined. This section relies heavily on similar developments in propeller cavitation noise research.
\end{abstract}

Keywords. Turbomachinery; cavitation; thermodynamic effects; noise.

\section{Introduction}

The problem of cavitation, which is local vaporization in liquid flows due to reduction in static pressure, and its association with turbomachinery, is an old one. In general, cavitation is to be avoided since it can result in material damage, loss in performance, inducement of vibration and intense noise radiation over a wide frequency band. In some special applications, another deleterious effect due to cavitation could be the change of chemical composition of the original liquid sample. Therefore, it is understandable that considerable efforts are still being expended in trying to tackle the problem of cavitation in turbomachinery flows, since even the rudimentary problem of prediction of the conditions for the onset of some of the physical effects due to cavitation as noted above is far from solution.

One of the commonly used methods for assessing the potential occurrence of cavitation in a prototype turbomachine is through model-testing in a suitable facility. In order to conduct the tests properly and extrapolate the findings to prototype conditions, it is necessary to establish proper scaling laws. It is most convenient to do this through the use

A list of symbols is given at the end of the paper 
of non-dimensional parameters. In addition to the non-dimensional parameters associated with well-established affinity rules for the performance of turbomachines under noncavitating conditions, it is necessary to use an additional non-dimensional parameter when dealing with cavitating conditions. As indicated in well-known text books on the subject (for example, Wislicenus 1947) the relevant parameter most widely used is the Thoma cavitation parameter, defined by,

$$
\sigma=H_{s v} / H
$$

where, $H_{s v}$ is the appropriate pressure head above the vapour pressure head and $H$ is the difference in the head across the machine. In the case of pumps, $H_{s v}$ is commonly termed the Net Positive Suction Head (NPSH) and an expression for computing the same is

$$
H_{s u}=H_{s}+\left(V^{2} / 2 g\right)-H_{v} .
$$

Here, $H_{s v}$ is the absolute static pressure head at the inlet, $V$ is the average velocity at the inlet and $H_{v}$ is the vapour pressure head evaluated at the bulk liquid temperature at the inlet. In the case of a turbine, referring to figure $1, H_{S v}$ is given by

$$
H_{s v}=H_{a}+h_{s}-H_{v},
$$

where $H_{a}$ is the atmospheric pressure head; here, velocity effects have been neglected. It should be clear that the magnitude of $\sigma$ can be specified irrespective of whether cavitation occurs or not in the machine. Hence, the magnitude of $\sigma$ in a given situation is referred to as the plant $\sigma$ and its relation to a critical $\sigma$ is then a measure of the extent of cavitation. For this purpose, we can define three specific values of $\sigma$ and denote them as $\sigma_{i}, \sigma_{c}$ and $\sigma_{b}$ with their relevance indicated below.

$\sigma_{i}-$ This is the value of $\sigma$ at which the first signs of cavitation are detected. Therefore, the most sensitive method of detecting cavitation should be used to infer $\sigma_{i}$ and experience suggests that monitoring noise levels at high frequencies could be one such method.

$\sigma_{c}-$ This is the value of $\sigma$ at which the first signs of performance deterioration of the machine due to cavitation become apparent. One cannot be precise about this measure and some arbitrariness would be essential in arriving at a value of $\sigma_{c}$. The commonly used measure of $3 \%$ head drop from non-cavitating conditions is one method used frequently in centrifugal pump applications.

$\sigma_{b}$ - This is the value of $\sigma$ at which breakdown in the performance of the machine due to cavitation occurs.

From the criterion indicated, we expect $\sigma_{i}>\sigma_{c}>\sigma_{b}$ and if $\sigma>\sigma_{i}$ no cavitation is expected. The difference in $\sigma_{i}$ and $\sigma_{c}$ values can differ from machine to machine; for

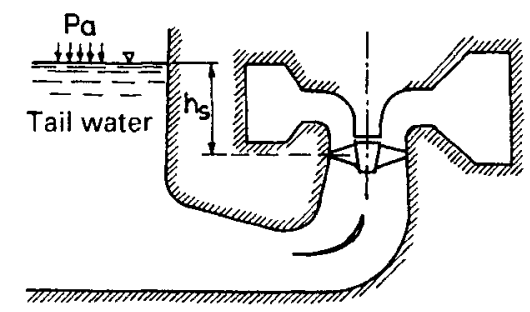

Figure 1. Figure illustrating parameters in computing suction head for a turbine. 
example, McNulty \& Pearsall (1979) indicate values of 2 to 8 for the ratio of inception to critical cavitation number for different pumps. In their experiments, inception value was arrived at by an acoustic method of detection and critical value was based on 3\% head drop criterion. The definitions for $\sigma_{i}$ and $\sigma_{h}$ are less ambiguous as compared to that for $\sigma_{r}$. Several possibilities exist for the latter and the implications of these in practical use have, for example, been discussed by Schiele et al (1974). However, it should be noted that a distinction between $\sigma_{i}$ and $\sigma_{c}$ is not always made.

The cavitation number, $\sigma$, defined above is only one of the several definitions used in practice. A complete list of the several variations in the definition of $\sigma$ for centrifugal pumps is given by Kumaraswamy (1986). It is not the purpose here to indicate the preferred definition for $\sigma$; however, we would like to introduce another cavitation number, $K$, which is useful in relating cavitation in the machine to the cavitation properties of individual vane or blade sections. This number, primarily useful for pumps is defined as,

$$
K=\left(P_{s-} P_{n}\right) / \frac{1}{2} \rho\left[V^{2}+L^{2}\right] \text {. }
$$

where $P_{s}$ refers to the static pressure and $V$ is the axial velocity at the inlet of the pump, $U$ the tip velocity equal to $\pi D n$ and $P_{z}$ the vapour pressure at upstream bulk temperature. Further, $K_{i}, K_{c}$ and $K_{b}$ are defined in the same manner as $\sigma$ and have similar physical significance. Another possibility is to replace the denominator of (4) by the dynamic head based on relative velocity at pump inlet. This will be particularly applicable when there are inlet guide vanes.

If cavitation number is the only additional significant parameter, then we can expect complete similarity in the relative extent of cavitation etc. in geometrically similar machines at equal $\sigma$ values, provided the operation points of the machine are fixed by the classical affinity laws. Any deviations from this can in general be termed as "scale effects". Several types of scale effects have been observed and are associated with various sources like viscous effects, thermodynamic effects etc. The scale effects seem to be most severe in determining the conditions for the onset of cavitation. Understanding the scale effects in general is one of the important topics of current cavitation research.

There are several aspects to the problem of cavitation in turbomachinery, the fundamental and most significant one being the ability to predict the conditions for the onset of cavitation in such machines. There are others, like assessing the potential loss in performance, extent of damage, levels of radiated noise etc. once cavitation sets in. In principle, it would be highly desirable to achieve this capability at the design stage itself. As noted earlier, we are far from such a possibility and it can be appreciated that for comprehensive coverage, in one article of the present type, some choice of topics is a necessity and presently three have been chosen. The first one is a summary of the recent developments in understanding the mechanism of cavitation inception. The second one concerns the thermodynamic effects in pump cavitation and the third one is on cavitation noise where emphasis is placed on presenting data from propeller cavitation noise measurements. It is believed that all the three topics are of current relevance.

\section{Recent developments in understanding the mechanism of cavitation inception}

\subsection{Background}

In general the two conditions which must be satisfied for the occurrence of cavitation are: (i) the availability of "nuclei" (the weak spots in the liquid), and (ii) the reduction of static 
pressure to or below the vapour pressure. Therefore, considerable effort has been expended in trying to understand the role of nuclei and viscous effects, which controls the local excursions in static pressure, in cavitation inception. In the following, we only provide a summary and the interested reader can consult several review articles on the subject like Arndt (1981), Acosta et al (1986), Arakeri (1986), Billet (1986) and Kuiper (1985) for additional information.

\subsection{Role of nuclei}

Since the tensile strength of a pure liquid is predicted and observed, in careful experiments, to be quite high, the only explanation for observed cavitation at moderate to negligible tensile strengths is due to the presence of weak spots in the liquid, commonly termed as nuclei. One excellent source and a viable model for nuclei is the undissolved gas bubble. Organic and inorganic particulates are also potential sources of nuclei. From static stability considerations of a gas bubble, it is a simple matter to show (see Knapp et al 1970) that the critical pressure required for unstable growth (indicating rapid vaporization or cavitation onset) is given by,

$$
P_{c}=P_{v} \frac{4 S}{3 R^{*}} .
$$

This expression is obtained by assuming the permanent gas in the bubble to expand or contract isothermally. In the above expression $P_{v}$ is the vapour pressure of the liquid, $S$ is the coefficient of surface tension $\left(0.072 \mathrm{~N} / \mathrm{m}\right.$ for air-water interface) and $R^{*}$ is the characteristic size of the nuclei. From (5), one can infer that the classical assumption of critical pressure being equal to the vapour pressure is accurate only in the presence of large sized nuclei, typically exceeding $100 \mu \mathrm{m}$. One possible method of overcoming scale effects associated with nuclei size would be to introduce $P_{c}$ instead of $P_{v}$ in (4). In such a case, it is clear that the non-dimensional contribution from the term containing nuclei of radius $R^{*}$ decreases with increase in the resultant or tip velocity and also that measurement or knowledge of nuclei size distribution would be required under prototype and model testing conditions. An excellent summary of the efforts in the measurement of nuclei size distribution is given by Billet (1986).

As indicated by Billet (1986), measurement of cavitation nuclei has been attempted using basically two different approaches. One is to measure the particulate/microbubble

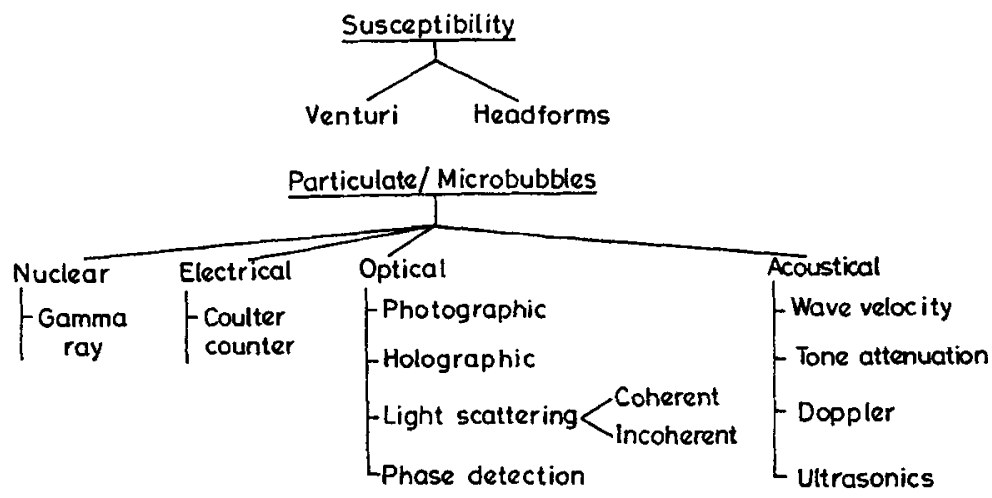

Figure 2. Cavitation nuclei measuring techniques (Billet 1986). 


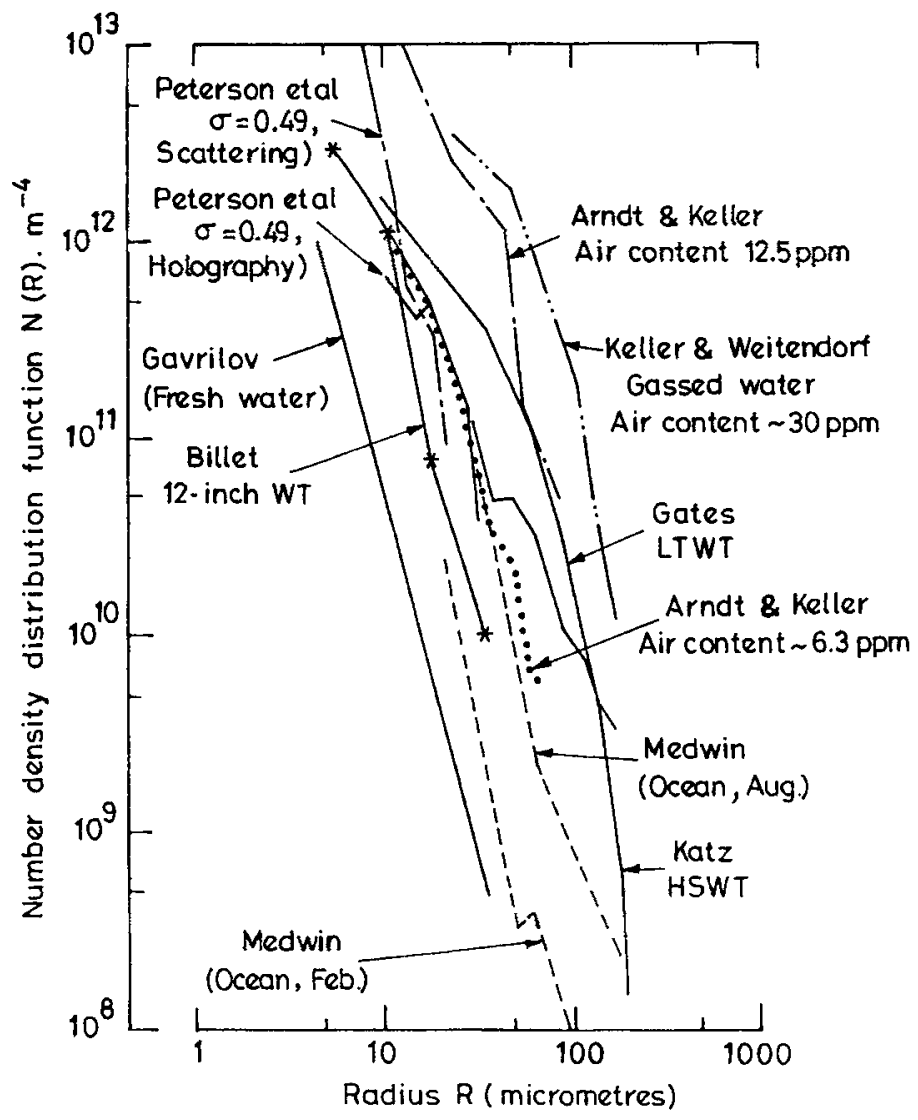

Figure 3. Compilation of microparticulate and microbubble number density distributions from various sources (Billet 1986).

distributions by utilizing acoustical, electrical or optical methods and the second is to establish the cavitation susceptibility of the liquid sample through direct cavitation monitoring. A list of the various cavitation nuclei measuring techniques is indicated in figure 2. Among these, the holographic technique gives a direct measure of particulate and microbubble distribution and hence has become the standard with which measurements with other methods are compared. Figure 3 gives a summary of some nuclei distribution data obtained by using various techniques. These nuclei distributions are expressed in terms of a number density function which is defined as

$$
\begin{aligned}
n(R) & =n\left\{\frac{R_{1}+R_{2}}{2}\right\} \\
& =\frac{\text { number of nuclei per unit volume with radii between } R_{1} \text { and } R_{2}}{R_{2}-R_{1}}
\end{aligned}
$$

and is plotted against nuclei radius in micrometres. These data in most cases represent both microbubble/particulate distributions and can be represented by the relationship

$$
n(R) R^{3}=\text { constant. }
$$


The practical utility of such measurements in either the prediction of the conditions for cavitation inception or improvements in scaling procedures is not yet established. However, just like measurement of free stream turbulence levels for wind tunnels, the measurement of nuclei size distribution will become standard practice for characterizing a given facility or environment.

In view of the difficulties associated with the direct utility of nuclei size distribution measurements, an alternate method of developing the so-called cavitation susceptibility meters (CSM) has been initiated. The most widely used method utilizes a venturi system (Oldenziel 1979; Lecoffre \& Bonin 1979), even though earlier, use of head forms was suggested (Silberman et al 1973). In the case of the former, a small venturi tube made of glass or steel is utilized and on adjusting the volume flow rate through the system, the minimum pressure varies. At a given minimum pressure, the number of cavitation events are recorded either optically or acoustically and thus can provide a direct measure of both the liquid sample critical pressure and nuclei concentration over a very large size range. More recently, a comparison of results from CSM with the nuclei distribution measured with holography has been made by d'Agostino et al (1988). In addition to the above methods of nuclei measurement, the old technique of total gas (Van Slyke apparatus) or dissolved oxygen content monitoring continues and is routinely reported even though not to the extent desired.

\subsection{Viscous effects}

One of the first studies in relating the viscous effects to cavitation inception process is that due to Parkin \& Kermeen (1953). This classic study shows that the mechanism of cavitation inception process on a hemispherical nosed axisymmetric body is closely related to its boundary layer characteristics. Arakeri \& Acosta (1973) repeated their experiments with the use of the Schlieren technique of flow visualization. They found that cavitation inception first occurs in the reattachment zone of a laminar separation zone and well away from the theoretically predicted location of minimum pressure. Subsequent studies have confirmed the significant role of turbulent transition, whether in attached boundary layers or in shear layers in cavitation inception. At the present time, what is known is that the transition zone is the location of intense pressure fluctuations. For example, recent studies on free shear layers by Ooi \& Acosta (1984) and Arakeri \& Berman (1989) seem to indicate peak pressure fluctuation levels of the order of 160 and $400 \%$ of the dynamic head in these regions. There is no doubt that the low pressure zones detected are at the core of vortices which form in the initial zone of transition and subsequently get stretched in the strong zone of shear. The latter is a complex three-dimensional phenomenon and extremely difficult to analyse or probe. A testimony to this is the recent finding of Katz \& O'Hern (1983) that the first location of cavitation inception in a two-dimensional free shear layer is in vortices aligned roughly $45^{\circ}$ to the direction of mean flow and not in vortices aligned normal to the direction of mean flow. The latter are the readily visible structures in classical flow visualization techniques. In addition to the viscous effects being responsible for large pressure excursions in a given flow field, the presence of a separated region, which again is a consequence of viscous effect, could influence cavitation inception differently. These zones, being characterized by large time scales compared to the main flow, could be regions of nuclei size modification through mass transfer across the walls of original nuclei being entrained in this zone. Many of these issues are still being investigated. 


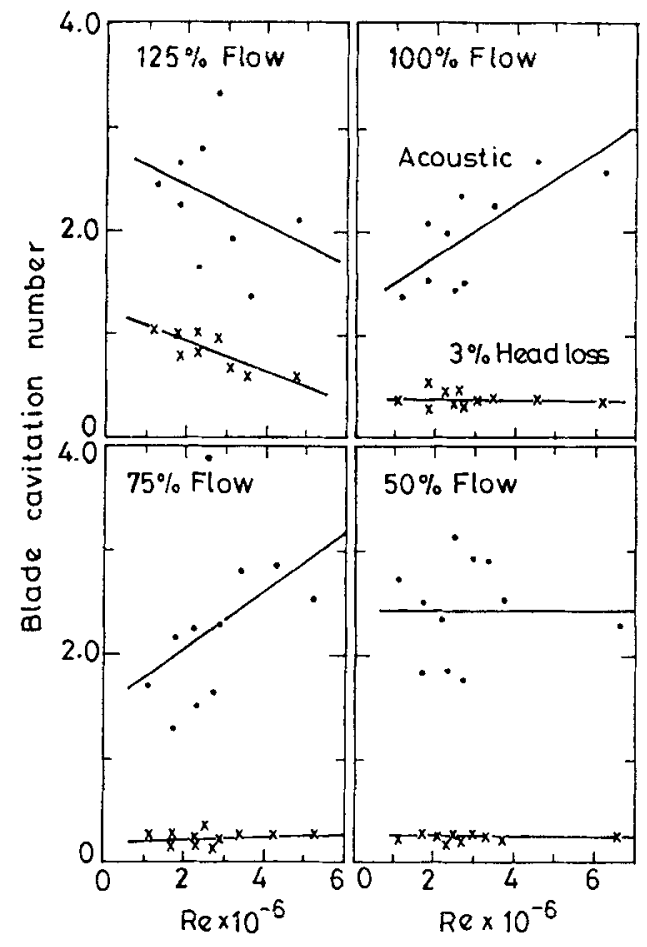

Figure 4. Variation of inception and critical cavitation numbers with Reynolds number (McNulty \& Pearsall 1979).

\subsection{Relevance to turbomachinery}

In going through the literature on cavitation inception problems in turbomachinery, it is found that hardly any reference is made to the recent advancements in understanding the mechanism of cavitation inception and some of its aspects that were indicated above. On the other hand, many of the findings have been exploited usefully to understand the mechanism of cavitation inception on marine propellers, flow around which is similar to that of a turbomachine. A summary of these efforts is contained in the work of Kuiper (1981). The reason perhaps is in the fact that in turbomachinery applications, it is very rarely that one is interested in the beginning or incipient conditions of cavitation; the parameter of more interest being $\sigma_{i}$ rather than $\sigma_{i}$. There is no doubt that the conditions for $\sigma_{i}$ are critically dependent on detailed features of flow like nuclei content and modification of pressure fields due to viscous effects. This aspect is clear from the work of McNulty \& Pearsall (1979) and is shown in figure 4. The Reynolds number effect is clearly more dominant in determining the conditions for inception cavitation number based on acoustic detection rather than on critical cavitation number based on $3 \%$ head drop criterion.

With the advent of powerful computers and advances in modeling of turbulent flows, it is becoming easier to compute the real flow field in a turbomachine numerically. These should be of some help in determining the primary pressure fields associated with such flows and hence conditions for cavitation associated with classical minimum pressures. However, with boundary layers and lifting surfaces, turbomachinery flow is prone to generating locally strong vortical flows. Minimum pressures associated with these 
secondary pressure fields cannot be predicted with sufficient accuracy since in many cases even the existence of such vortices may not be predicted. In this connection, it should be noted that computation of pressure fields in the tip-vortex region of a lifting hydrofoil is still a challenging task. It is most likely that cavitation inception in unexpected regions of turbomachine flow is due to formation of local vortices. Some examples of such observations have been given by Couston et al (1987), who also indicate how judicious use of computation helps in alleviating the difficulties. The point to be made here is that the fundamental investigations on cavitation inception could be of considerable significance in understanding cavitation inception not associated with the primary pressure fields and some of these could even be eliminated with minor modifications.

Investigations on potential scale effects associated with nuclei content of the liquid and viscous effects in turbomachinery flows have assumed added significance, since the acoustic technique is increasingly being mentioned as a possible candidate for "condition monitoring" of turbomachines including the effects of cavitation. One of the physical effects which is most sensitive to cavitation onset is the sudden increase in radiated noise levels, in particular, at higher frequencies. Therefore, with acoustic monitoring, the conditions first detected are those associated with cavitation inception or the parameter $\sigma_{i}$. This is bound to lead to difficulties in extrapolating model test results to prototype situations unless design improvements are made to ensure that minimum pressures are associated with primary pressure fields and not the secondary, this being particularly so for off-design operating conditions. The crucial input to these efforts will come only from fundamental investigations on cavitation inception in turbomachinery flows based on similar work done in other classes of flows, like flow past axisymmetric bodies or individual lifting hydrofoils (Van der Muelen 1980).

\section{Thermodynamic effects in pump cavitation}

\subsection{Background}

Since cavitation is a process of vaporization resulting in vapour bubbles or regions, a certain amount of heat to cater to the required latent heat of vaporization has to be supplied by a thin layer of liquid surrounding the bubble or region. This necessarily involves a temperature drop or depression in the liquid surrounding the vapour region and a corresponding vapour pressure difference can be evaluated using the Clausius-Clapeyron equation. Now, considering a cavitating pump we can define two cavitation numbers $\sigma$ and $\sigma_{L}$ as follows,

$$
\sigma=\frac{H_{s}+\left(V^{2} / 2 g\right)-H_{v}\left(T_{\infty}\right)}{H}=\frac{\mathrm{NPSH}}{H}
$$

and

$$
\sigma_{L}=\frac{H_{s}+\left(V^{2} / 2 g\right)-H_{v}\left(T_{L}\right)}{H}=\frac{\mathrm{NPSH}_{L}}{H},
$$

where $T_{\infty}$ is the bulk temperature at the inlet and $T_{L}$ is the average local temperature at the surface of the vapour region and the difference between the two is the temperature drop mentioned earlier. We can express $\sigma$ in the form

$$
\sigma=\sigma_{L}-\Delta \sigma
$$


where $\Delta \sigma$ is given by

$$
\Delta \sigma=\frac{H_{v}\left(T_{\infty}\right)-H_{v}\left(T_{L}\right)}{H}=\frac{\Delta H_{v}}{H} .
$$

In water, near room temperature the term $\Delta \sigma$ is negligible and hence $\sigma \approx \sigma_{L}$ and one need not distinguish at what temperature the vapour pressure head is to be evaluated. However, this need not be the case for water at higher temperatures and in particular for other fluids like hydrocarbons or cryogenics. In this case we may not expect similarity on the basis of $\sigma$ alone and this may be attributed to the thermodynamic scale effect, since, the origin of $\Delta \sigma$ is primarily associated with thermal effects. Experiments like those of Holl et al (1975) and Hutton \& Furness (1974) show that cavitation similarity is observed on the basis of equal values of $\sigma_{L}$ and not $\sigma$, under conditions where thermodynamic effects are significant.

On the basis of the above considerations we can arrive at qualitative implications of thermodynamic effects in pump cavitation. Consider a pump operating in cold water with a flow rate of $Q_{1}$, and developing a head of $H_{1}$ under non-cavitating conditions; it requires an NPSH of (NPSH) $)_{1}$ to result in $3 \%$ head drop due to cavitation. The same pump operates in hot water or another fluid at a flow rate of $Q_{1}$ and develops the same head $H_{1}$ (this tacitly assumes that non-cavitating performance is not influenced by thermodynamic effects); the question is what NPSH denoted by (NPSH) 2 would be required to result in $3 \%$ head drop. It has been stated that cavitation similarity is expected on the basis of equal local cavitation numbers or $\left(\sigma_{L}\right)_{1}=\left(\sigma_{L}\right)_{2}$. This implies

$$
\sigma_{1}+\Delta \sigma_{1}=\sigma_{2}+\Delta \sigma_{2}
$$

and, since $\Delta \sigma_{1}$ is zero, we get

$$
\sigma_{1}-\sigma_{2}=\frac{(\mathrm{NPSH})_{1}-(\mathrm{NPSH})_{2}}{H_{1}}=\frac{\Delta(\mathrm{NPSH})}{H_{1}}=\Delta \sigma_{2}=\frac{\left(\Delta H_{v}\right)_{2}}{H_{1}} .
$$

Therefore, the NPSH correction or, as Stepanoff (1965) prefers to call it, NPSH adjustment is directly related to $\Delta H_{v}$ associated with thermodynamic effects. Further, we expect the NPSH performance in the second case to be better, in the sense the NPSH required is lower than that of cold water. Thus, the focus of efforts in assessing thermodynamic effects in pump cavitation is to arrive at a method of predicting $\Delta H_{v}$.

\subsection{Conventional criterion and methods}

The most widely used method for assessing thermodynamic effects in pump cavitation is via the so-called $B$ factor method proposed by Stepanoff (1961). The starting point for this method is the heat balance written in the neighbourhood of the cavity surface and has the form,

$$
\rho_{v} \underline{V}_{\eta} \lambda=\rho_{l} \underline{V}_{l} C_{l} \Delta T
$$

where $\underline{V}_{v}$ and $\underline{V}_{l}$ are the volume of vapour and liquid respectively. The $B$ factor is, by definition, the ratio of the vapour volume to the liquid volume or

$$
B=\frac{\underline{V}_{v}}{\underline{V}_{l}}=\frac{C_{l} \Delta T}{\lambda} \frac{\rho_{l}}{\rho_{v}} .
$$


From the above, we can relate the temperature depression, $\Delta T$ to $B$ and using the ClausiusClapeyron equation the corresponding $\Delta H_{v}$ is given by

$$
\Delta H_{v}=J\left[\frac{\rho_{v}}{\rho_{l}}\right]^{2}\left[\frac{\lambda^{2}}{C_{l} T}\right]\left[\frac{g_{c}}{g}\right] B
$$

Stepanoff \& Kowaguchi (1962) define a new parameter $B_{1}$ related to $\Delta H_{v}$ and $B$ by

$$
B=B_{1} \Delta H_{v},
$$

and on the basis of experimental data provide an "equation of state"

$$
B_{1}^{4 / 3} \Delta H_{v}=64 / H_{v}
$$

to compute $\Delta H_{v}$ directly in terms of $B_{1}$, which can be evaluated from the thermodynamic properties of the liquid and vapour at the bulk temperature and $H_{v}$ which also can be obtained. They provide a ready graph to evaluate NPSH adjustment for hydrocarbons based on the $B$-factor method. One criticism of the $B$-factor method is that it does not involve any dynamic terms which are likely to be significant in determining heat transfer rates at the interface. The importance of the POGO instability in the operation of liquid propellant rockets (Rubin 1966), and the role played by the fuel and oxidizer turbo pumps in that instability, generated renewed interest in analysing thermodynamic effects in pump cavitation handling cryogenic fluids. Two groups have been active in these efforts; one at the Pennsylvania State University (Holl et al 1975), and the second at NASA Lewis Research Center (Ruggeri \& Moore 1969); the noted references are representative. An analysis based on an energy balance at the liquid vapour interface including convective effects, the group from the Pennsylvania State University propose the following expression for the temperature depression, $\Delta T$

$$
\Delta T=\frac{C_{Q}}{C_{A}} \cdot \frac{\mathrm{Pe}}{\mathrm{Nu}} \cdot \frac{\rho_{v}}{\rho_{l}} \cdot \frac{\lambda}{C_{l}} .
$$

Here, $C_{Q}$ is a flow coefficient to cater to the vapour entrainment at the end of an attached cavity, $C_{A}$ is an area coefficient that includes geometrical features of the cavity. Extensive experimental studies were conducted using venturies and ogive head forms with several cryogenics as working fluids to provide correlations for evaluating $C_{Q}, C_{A}, \mathrm{Pe}$ and $\mathrm{Nu}$. To the best of our knowledge, the above correlations have not been tested for assessing thermodynamic effects in pump cavitation.

Based on theoretical analysis and coupled with experimental venturi cavitation results, the NASA Lewis Research Center group, propose the following expressions to predict $B$,

$$
B=B_{R}\left[\frac{\alpha_{R}}{\alpha}\right]^{1.0}\left[\frac{V}{V_{R}}\right]^{0.8}\left[\frac{D}{D_{R}}\right]\left[\left(\frac{\Delta x}{D}\right) /\left(\frac{\Delta x}{D}\right)_{R}\right], \quad \text { in general }
$$

and

$$
B=B_{R}\left[\frac{\alpha_{R}}{\alpha}\right]^{1.0}\left[\frac{n}{n_{R}}\right]^{0.8}, \quad \text { for a particular pump. }
$$

Here, subscript $R$ refers to reference values and once $B$ is evaluated, the corresponding $\Delta H_{v}$ can be obtained from (16). Ruggeri \& Moore (1969) have used the above correlation quite 
successfully to predict thermodynamic effects in pumps and inducers. However, the use of the correlation requires that two sets of appropriate test data be available for each pump and operating conditions of interest, and at least one set of data must provide a measurable thermodynamic effect. The latter is a distinct disadvantage of the method, but what is of relevance is that the test data need not necessarily be for the same liquid for which thermodynamic effects are to be predicted. Thus, easy to handle liquids that exhibit thermodynamic effects could be used to generate the test data.

There are other methods which have been suggested and developed for predicting thermodynamic effects in pumps like those due to Spraker (1965), Chivers (1969-70), Bonnin (1974) etc: however, these also involve parameters similar to those indicated above.

\subsection{Possible new criterion and correlation}

In the following, we first develop a new criterion which is based on the early work of Plesset (1964) and extended later by Brennen (1973). For this purpose, consider cavitating flow past a blunt circular cylinder (also known as zero-caliber ogive) as depicted in figure 5 . The non-cavitating flow past the body and the incipient cavitation condition are characterized by the minimum pressure coefficient, $C p_{\min }$ and the incipient cavitation number, $K_{i}$. Under steady state conditions, the heat balance at the cavity surface requires,

$$
\lambda \underline{m}_{v}=h\left(T_{x}-T_{L}\right) A_{s} .
$$

Here, $\underline{m}_{\eta}$ is the measure of the rate of vapourization and $A_{s}$, the interface cavity surface area. The heat transfer coefficient, $h$, can be written as

$$
h=k_{l} / \delta_{t},
$$

with $\delta_{t}$ being the thermal boundary layer thickness and evaluated as,

$$
\delta_{t}=\left(\alpha_{l} \tau\right)^{1 / 2}
$$

where $\tau$ is a relevant time scale.

Based on the fact that the time scale could be associated with the extent of cavitation, we propose the following expression for $\tau$,

$$
\tau=\left(L / U_{\infty}\right)\left(\left|C p_{\min }\right| / K_{L}\right)
$$
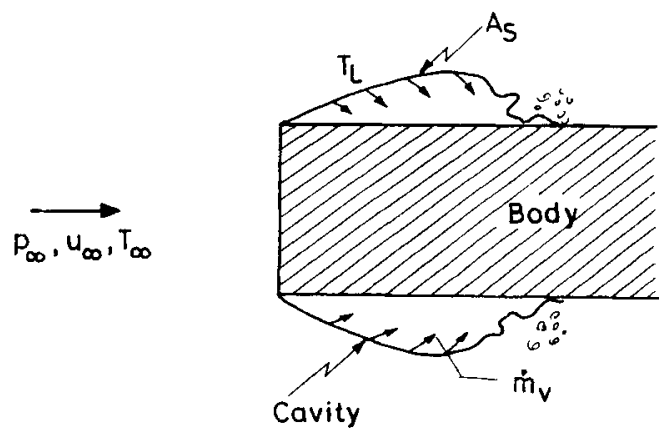

Figure 5. Schematic of cavitating flow past a blunt circular cylinder (zero-calibre ogive). 
where $K_{L}$ is the local cavitation number and $L$ is the characteristic body dimension. The introduction of $\left|C p_{\min }\right|$ and $K_{L}$ in addition to the normally expected time scale of $L / U_{\infty}$ is consistent with the fact that $\tau$ increases with increase in the magnitude of $\left|C p_{\min }\right|$ at a fixed value of $K_{L}$ and at a fixed value of $\left|C p_{\min }\right|$ it also increases with decrease in the magnitude of $K_{L}$. The basic heat balance relationship can now be expressed as,

$$
\lambda\left(\frac{\underline{m}_{v}}{A_{s}}\right)=\frac{k_{l}}{L}\left(\frac{U_{\infty} L}{\alpha_{i}}\right)^{1 / 2}\left[\frac{K_{l}}{\left|C p_{\min }\right|}\right]^{1 / 2}\left(T_{\infty}-T_{L}\right) .
$$

From dimensional considerations it follows that,

$$
\underline{m}_{v} / A_{s} \approx \rho_{v} U_{\infty}
$$

and using further approximations of

$$
\begin{aligned}
K_{i} & \sim\left|C p_{\min }\right| \text { and } \\
K_{L} & \sim K
\end{aligned}
$$

we get for non-dimensional temperature depression,

$$
\frac{T_{\infty}-T_{L}}{T_{\infty}}=\left(\frac{\lambda}{C_{p_{l}} T_{\infty}}\right)\left(\frac{\rho_{v}}{\rho_{l}}\right)\left(\frac{U_{\infty} L}{\alpha_{l}}\right)^{1 / 2}\left[\frac{K_{i}}{K}\right]^{1 / 2} C_{0},
$$

where $C_{0}$ is a proportionality constant.

The integration of the Clausius-Clapeyron equation with the assumption that $\rho_{l} \gg \rho_{v}$ and the use of normal equation of state for the vapour results in

$$
\Delta H_{v}=\frac{P_{v}\left(T_{\infty}\right)-P_{v}\left(T_{L}\right)}{\rho_{l} g}=\frac{\rho_{v}}{\rho_{l}} \frac{\bar{R}}{M_{v}} \frac{T_{L}}{g}\left\{\exp \left[\bar{\lambda} \frac{M_{v}}{\bar{R}}\left(\frac{T_{\infty}-T_{L}}{T_{\infty} T_{L}}\right)\right]-1\right\} .
$$

The temperature difference appearing in the above expression is given by (28). This exact form should be used for cryogenics like liquid hydrogen, whose properties vary very strongly with temperature. However, in most other cases a valid approximation

$$
\exp \left[\bar{\lambda} \frac{M_{v}}{\bar{R}} \frac{\left(T_{\infty}-T_{L}\right)}{T_{\infty} T_{L}}\right]-1 \approx \bar{\lambda} \frac{M_{v}}{\bar{R}}\left[\frac{T_{\infty}-T_{L}}{T_{\infty} T_{L}}\right]
$$

should be quite accurate. Thus, with this approximation the expression for $\Delta H_{v}$ is,

$$
\Delta H_{v}=C_{0}\left[\frac{\lambda^{2}}{T C p_{l} g}\right]\left[\frac{\rho_{v}}{\rho_{l}}\right]^{2}\left(\frac{U_{\infty} L}{\alpha_{l}}\right)^{1 / 2}\left[\frac{K_{i}}{K}\right]^{1 / 2} .
$$

In non-dimensional form, the above can be expressed as

$$
\frac{\Delta H_{v}}{U_{\infty}^{2} / 2 g} \approx C_{0} \Sigma(T) \Lambda^{-1}\left[K_{i}\right]^{1 / 2}
$$

where the thermodynamic parameter $\Sigma(T)$ and the dynamic parameter $\Lambda$ are defined as

$$
\begin{aligned}
\Sigma(T) & =\left[\frac{\lambda^{2}}{T C p_{l} \sqrt{\alpha_{l}}}\right]\left[\frac{\rho_{v}}{\rho_{l}}\right]^{2}, \\
\Lambda & =\left(\frac{U_{\infty}^{3} K}{L}\right)^{1 / 2} .
\end{aligned}
$$


In (32), $\Sigma(T)$ is a non-dimensional measure of the thermodynamic effect and similarly $K_{i}$ is a measure of tendency for cavitation through pressure drop and therefore we could state that if thermodynamic effects are to be important, then

$$
\frac{\Delta H_{v}}{U_{\infty}^{2} / 2 g} \approx K_{i}^{1 / 2},
$$

which clearly is possible if

$$
\Sigma(T)>\Lambda
$$

Brennen (1973) using a different approach has arrived at the identical criterion.

For pumps, we can compute $\Lambda$ from

$$
\Lambda=\left(\frac{U^{3} \sigma}{D}\right)^{1 / 2}
$$

where $U$ is the tip velocity, $\sigma$ is the Thoma cavitation number and $D$ is the diameter of the impeller. Judicious choice of $U$ and $D$ in (36) may help improve correlations unless it is limited to the same design of pump. One may expect improved correlations if $U$ refers to the inlet relative velocity or preferably maximum relative velocity within the pump and $D$ is replaced by reference blade chord. These points need to be examined carefully.

For a typical fluid, like water, the qualitative behaviour of $\Sigma(T)$ versus $T$ is as shown in figure 6. For a given pump, $\Lambda$ can be computed and marked as shown in figure 6; at temperature $T=T^{*}, \Sigma(T)$ is equal to $\Lambda$ and our new criterion involves the following. For $T<T^{*}$, we do not expect any thermodynamic effects and for $T>T^{*}$, we look for a correlation of the form

$$
\Delta H_{v}=f\left(T_{R}\right),
$$

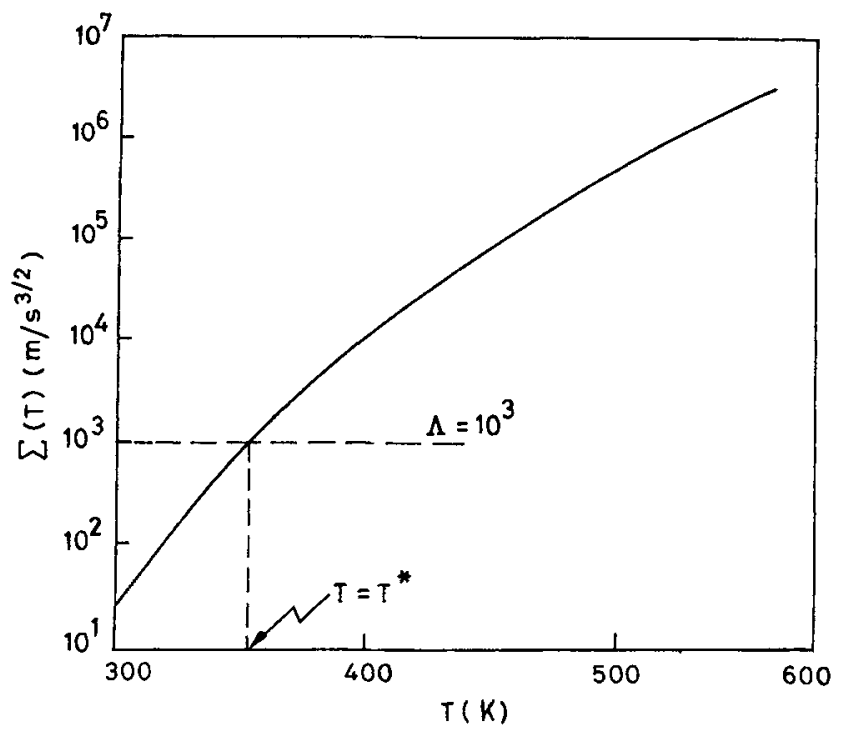

Figure 6. Qualitative behaviour of thermodynamic parameter, $\Sigma$, with temperature. 
where $T_{R}$ is the reduced temperature given by

$$
T_{R}=\frac{T-T^{*}}{T_{c}-T^{*}},
$$

with $T^{*}$ already defined, $T_{\mathrm{c}}$, is the critical temperature of the fluid under consideration and a tabulated quantity.

\subsection{Verification using new correlation}

The use of the correlation first of all requires the computation of $\Sigma(T)$ that can be done with the help of (33) and appropriate property tables. The computed values of $\Sigma(T)$ for various fluids as a function of temperature, $T$, are given in table 1 . Next, we look for a correlation of the form $\Delta H_{v}=f\left(T_{R}\right)$ from the existing pump cavitation data involving different fluids. The test data used along with relevant parameter values are summarized in tables 2 and 3. It should be noted that six different pumps and various fluids are involved. The $\Delta H_{n}$, values indicated in the table refer to the difference in the NPSH value for $3 \%$ head drop involving thermodynamic effect and the NPSH value corresponding to cold water data. The $T^{*}$ values were obtained for each of the pumps and the fluids by using the criterion that at $T=T^{*}, \Sigma(T)=\Lambda$. A plot of $\Delta H_{\|}$versus $T_{R}$ for all the six pumps with water as working fluid is shown in figure 7 . It should be noted that the data correlates very well, in particular keeping in mind that the uncertainty in the measurement is $\pm 0.5 \mathrm{ft}$ $( \pm 0.152 \mathrm{~m}$ ), the extent of which is shown in figure 7 . What is impressive is that the

Table 2. Summary of pump data used for correlation.

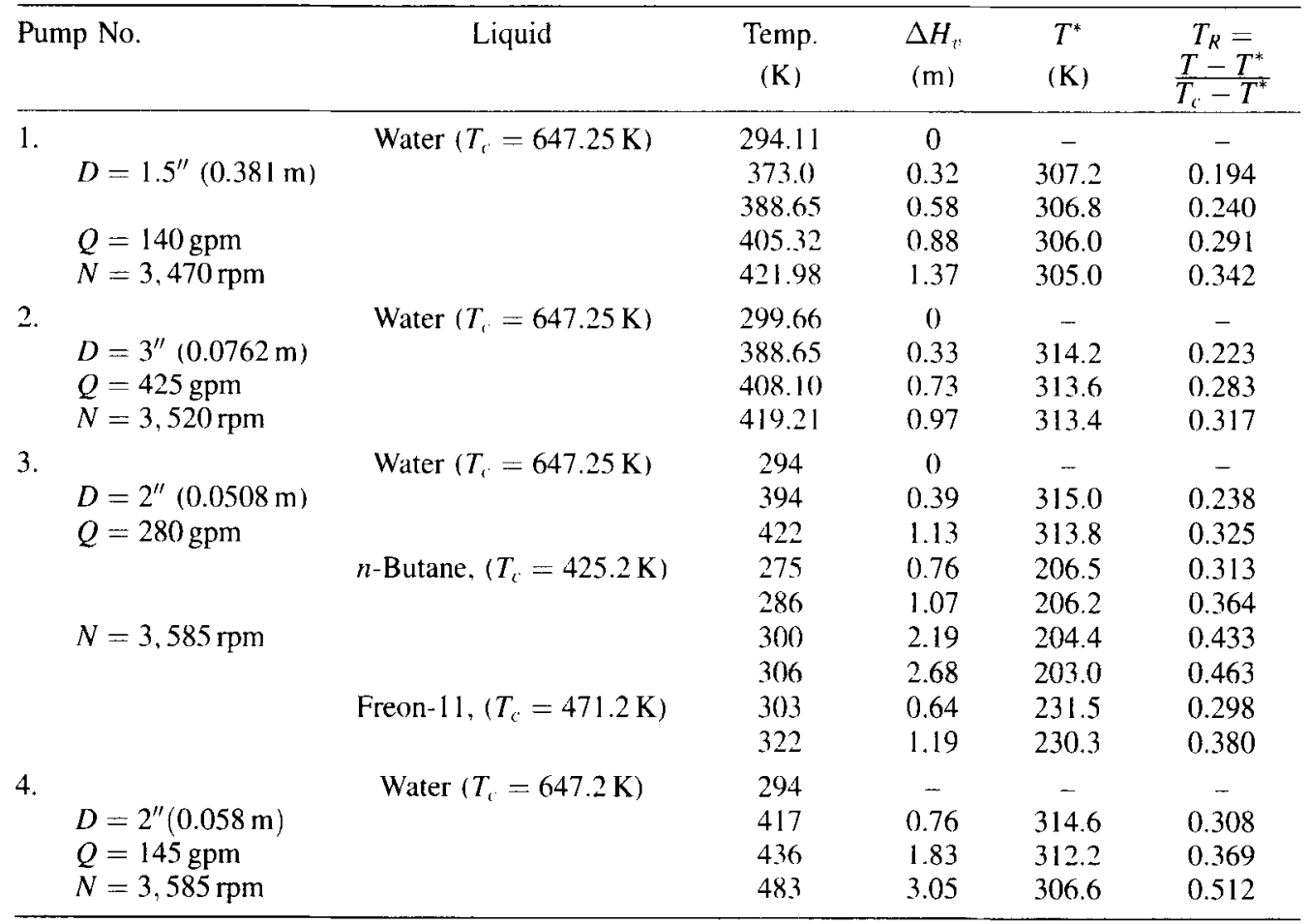

Source: Salemann (1959) and Stepanoff (1961) 
Table 3. Summary of pump data used for correlation.

\begin{tabular}{|c|c|c|c|c|c|}
\hline Pump No. & Liquid & $\begin{array}{l}\text { Temp. } \\
(\mathrm{K})\end{array}$ & $\begin{array}{l}\Delta H_{v} \\
(\mathrm{~m})\end{array}$ & $\begin{array}{l}T^{*} \\
(\mathrm{~K})\end{array}$ & $\begin{array}{l}T_{R}= \\
\frac{T}{T}-T^{*} \\
T_{c}-T^{*}\end{array}$ \\
\hline $\begin{aligned} D & =3^{\prime \prime}(0.0762 \mathrm{~m}) \\
Q & =600 \mathrm{gpm} \\
N & =3,550 \mathrm{rpm}\end{aligned}$ & $\mathrm{CH}_{3} \mathrm{OH}\left(T_{c}=512.7^{\circ} \mathrm{K}\right)$ & $\begin{array}{l}297 \\
333 \\
372 \\
394 \\
408 \\
420 \\
311 \\
339 \\
356 \\
368\end{array}$ & $\begin{array}{l}- \\
0 \\
0 \\
0.15 \\
0.46 \\
1.28 \\
0 \\
0.24 \\
0.61 \\
1.22\end{array}$ & $\begin{array}{c}- \\
318.4 \\
318.4 \\
318.2 \\
317.8 \\
317.0 \\
277.4 \\
277.2 \\
276.2 \\
276.2\end{array}$ & $\begin{array}{c}- \\
0.044 \\
0.163 \\
0.230 \\
0.274 \\
0.312 \\
0.143 \\
0.262 \\
0.336 \\
0.388\end{array}$ \\
\hline $\begin{array}{l}D=6^{\prime \prime}(0.1524 \mathrm{~m}) \\
Q=900 \mathrm{gpm} \\
N=1,785 \mathrm{rpm}\end{array}$ & $x^{2}$ & $\begin{array}{l}344.66 \\
382.2 \\
395.22 \\
408.00 \\
421.88 \\
327.44 \\
344.66 \\
361.33\end{array}$ & $\begin{array}{l}{ }^{-} \\
0.46 \\
0.70 \\
1.37 \\
0 \\
0.39 \\
1.01\end{array}$ & $\begin{array}{c}- \\
312.4 \\
311.2 \\
310.2 \\
306.6 \\
272.0 \\
271.2 \\
269.2\end{array}$ & $\begin{array}{c}- \\
0.202 \\
0.250 \\
0.290 \\
0.338 \\
0.230 \\
0.304 \\
0.378\end{array}$ \\
\hline
\end{tabular}

$* \mathrm{CH}_{3} \mathrm{OH}$ - methyl alcohol

Source: Spraker (1965)

correlation is good even at the highest water temperatures involved which is not the case with the correlations of Spraker (1965) in the form $\triangle \mathrm{NPSH}=f(1 / B)$ and the $B$-factor method of Stepanoff \& Kawaguchi (1962). The straight line through the data of figure 7 can be represented by

$$
\Delta H_{v}=10\left(T_{R}-0.21\right) .
$$

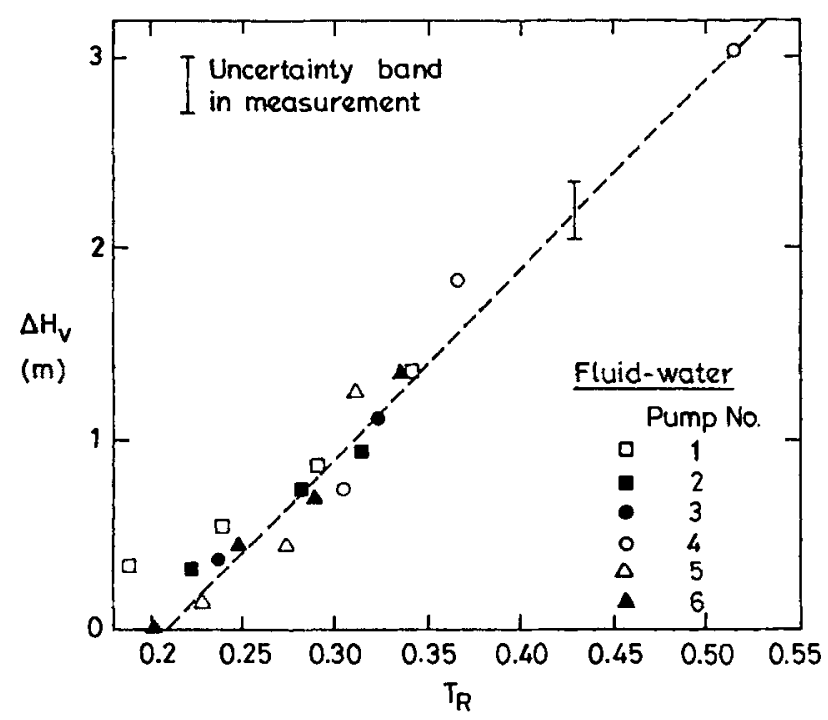

Figure 7. Correlation of observed vapour pressure head difference, $\Delta H_{v}$, with reduced temperature, $T_{R}$, for water. 


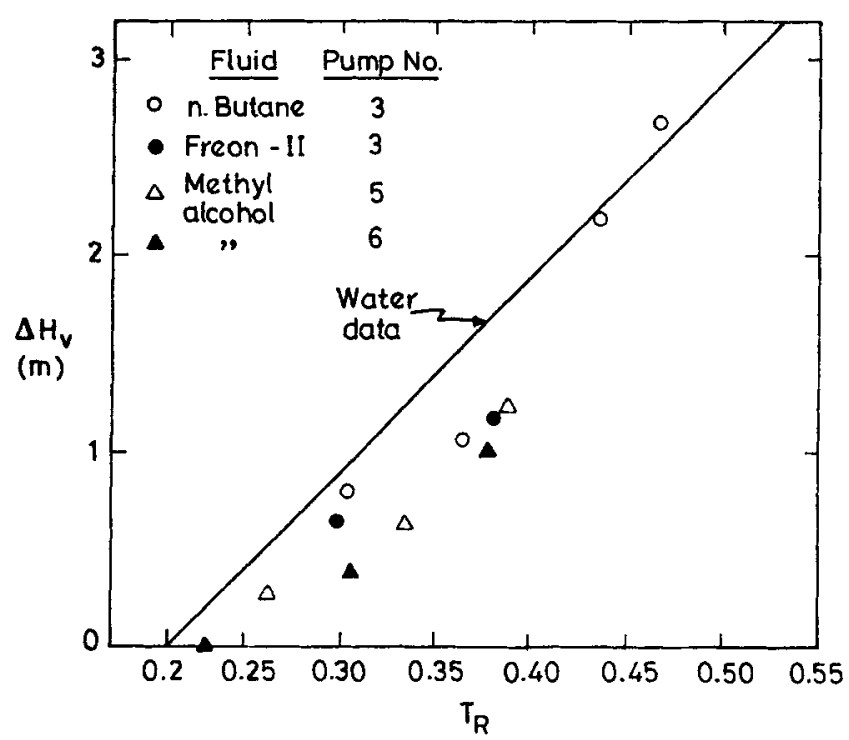

Figure 8. Correlation of observed vapour pressure head difference, $\Delta H_{i}$, with reduced temperature, $T_{R}$, for fluids other than water.

The above correlation is tested against observed $\Delta H_{v}$ data for some other pure liquids in figure 8 . In general, the $n$-butane data agree very well with the correlation; however, the methyl alcohol data are significantly lower and the refrigerant data follow the $n$-butane trend at a low $T_{R}$ value but methyl alcohol trend at a high $T_{R}$ value. It may be possible that the dissolved gas content may be a significant parameter for these other fluids. In any case, based on the water data we propose the following correlations for predicting NPSH adjustment, $\triangle$ NPSH in the terminology of Stepanoff.

$$
\begin{aligned}
& \triangle \mathrm{NPSH} \text { (in metres of liquid) }=0, \text { if } 0<T_{R} \leq 0.21, \\
& \triangle \mathrm{NPSH} \text { (in metres of liquid) }=10\left(T_{R}-0.21\right), \text { if } 0.21<T_{R} \leq 0.55 .
\end{aligned}
$$

\subsection{Remarks}

(1) The extent of thermodynamic effects varies from fluid to fluid. For example, the thermodynamic parameter $\Sigma$ at all working temperatures is high for liquid hydrogen suggesting that it is likely to "boil" rather than cavitate.

(2) The proposed new correlation for NPSH adjustment based on reduced temperature criterion predicts thermodynamic effects very well for water and reasonably well for other pure liquids.

(3) The new correlation is as simple to use as Stepanoff's and Spraker's methods but has the advantage that it includes dynamic effects in an indirect manner.

(4) For some liquids, it may not be possible to obtain $T^{*}$ as proposed and in this case it is suggested that the triple point temperature, $T_{t}$, could be used in place of $T^{*}$ for computing $T_{R}$.

(5) The present criterion is arrived at by assuming that the boundary layer is laminar at cavity interface: However, inclusion of turbulent diffusion effects, on the basis of the work of Brennen (1969), did not show encouraging results. 


\section{Cavitation noise}

\subsection{Background}

Cavitation can occur in various forms in a turbomachine, like bubble cavitation in blade surface low pressure regions, attached sheet cavitation at the leading edges of vanes or blades, tip clearance cavitation and so on; however, all of these involve some extent of bubble activity with bubble growth and collapse phases. In addition, developed cavities could be unsteady and thus cavitation can be a source of volume fluctuations in the fluid. This can result in radiated sound fields, which is generally random in nature and is termed cavitation noise. Per se, the presence of cavitation noise has not been of direct interest to designers of turbomachinery. However, as noted earlier, monitoring cavitation noise is now being thought of as a diagnostic tool to detect the onset of cavitation and also to assess potential cavitation damage in turbomachines. As pointed out by Robertson (1969), among the various effects due to cavitation, the one that becomes noticeable at the earliest stages is the dramatic increase in the level of radiated noise, in particular, at higher frequencies. This, at first, seems very attractive for cavitation detection since noise levels at high frequencies from other sources are relatively low in turbomachinery. However, there are difficulties (McNulty \& Pearsall 1979), like the location of cavitation is not known, which is only possible with visual detection. With improved instrumentation and signal processing (Courbiere et al 1982; Leggart \& Sponagle 1982; Martin \& Rao 1982) it may be possible to overcome some of the hurdles and it is felt that both basic and applied work should be pursued in correlating cavitation noise with extent of cavitation and damage. In this connection, it should be mentioned that the problem of cavitation noise has been of direct interest in naval applications like prediction and scaling of cavitation noise from marine propellers. This has led to several theoretical and experimental studies, which have been comprehensively dealt with by Blake (1986). We draw heavily from this source to consider certain aspects of cavitation noise in the following sections. First, however, in table 4 a summary of the various terms associated with characterizing cavitation noise is presented. For simplicity this is done for an ideal non-directional source of sound located at the centre of a spherical surface of radius $r$ and a surface area, $S_{A}$, of $4 \pi r^{2}$.

Table 4. Summary of various terms used in characterizing sound.

\begin{tabular}{llcc}
\hline Parameter and symbol & \multicolumn{1}{c}{ Definition } & Reference & Unit \\
\hline $\begin{array}{l}\text { Sound pressure } \\
\text { (root-mean-square; rms) } P\end{array}$ & $P=\left(P^{2}(f, \Delta f)\right)^{1 / 2}$ & - & $\mathrm{N} / \mathrm{m}^{2}$ or Pa \\
Sound intensity, $I$ & $I=P^{2} / \rho c$ & - & $\mathrm{W} / \mathrm{m}^{2}$ \\
Sound power, $W$ & $W=I S_{A}$ & - & $\mathrm{W}$ \\
Sound pressure level, $L_{P}$ & $L_{P}=20 \log \left(P / P_{\text {ref }}\right)$ & $P_{\text {ref }}=10^{-6} \mathrm{~N} / \mathrm{m}^{2}=1 \mu P a$ & $\mathrm{~dB} \mathrm{ref} 1 \mu \mathrm{Pa}$ \\
Sound intensity level, $L_{I}$ & $L_{I}=20 \log \left(I / I_{\text {ref }}\right)$ & $I_{\text {ref }}=10^{-12} \mathrm{Watt} / \mathrm{m}^{2}=1 \mathrm{pW} / \mathrm{m}^{2}$ & $\mathrm{~dB} \mathrm{ref} 1 \mathrm{p} \mathrm{W} / \mathrm{m}^{2}$ \\
Sound power level, $L_{W}$ & $L_{W}=20 \log \left(W / W_{\text {ref }}\right)$ & $W_{\text {ref }}=10^{-12}$ Watt $=1 \mathrm{pW}$ & $\mathrm{dB} \mathrm{ref} 1 \mathrm{pW}$ \\
\hline
\end{tabular}

Notes:

1. The definition of intensity $I$ and $W$ as indicated above are possible only under certain ideal conditions.

2. $\overline{P^{2}}(f, \Delta f)$ refers to measured mean square sound pressure at frequency $f$ and bandwidth $\Delta f$. Onethird-octave bandwidth is commonly used

3. In some applications, it is useful to convert sound pressure level to that which would exist if the measurements were carried out in $1 \mathrm{~Hz}$ band-width. This quantity is termed spectral density, SD, and is evaluated as $\mathrm{SD}=L_{P}-10 \log \Delta f$. It is expressed in $\mathrm{dB}$ ref $1 \mu \mathrm{Pa} / \mathrm{Hz}$. 


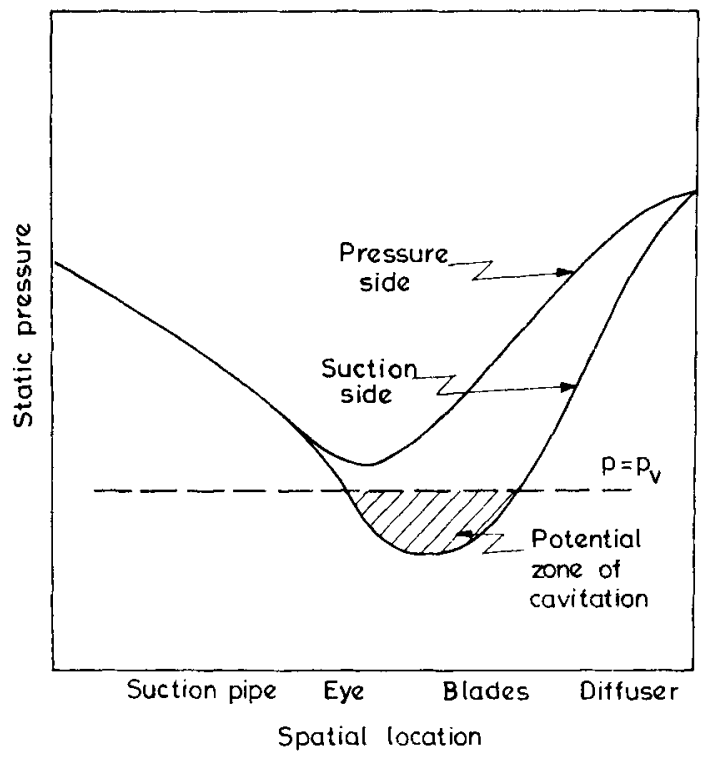

Figure 9. Schematic of the pressure field sensed by a cavitation nuclei in a pump.

\subsection{Single bubble cavitation noise and scaling law}

A schematic of the spatial pressure field sensed by a "cavitation nuclei" brought into the low pressure region of a pump is shown in figure 9 . This is similar to a pressure field for the case of a flow past a solid body as depicted in figure 10 and in both cases we expect a bubble growth and collapse pattern as illustrated in figure 10. If the growth and collapse could be considered spherically symmetric, then the radiated pressure field would be that from a monopole sound source and under certain assumptions, the expression for the radiated sound field from such a source in terms of acoustic pressure $P_{a}(r, t)$ is given by (see for example Blake 1986),

$$
P_{a l}(r, t)=\frac{\rho_{l}}{4 \pi} \frac{\ddot{Q}(t-r / c)}{r}
$$

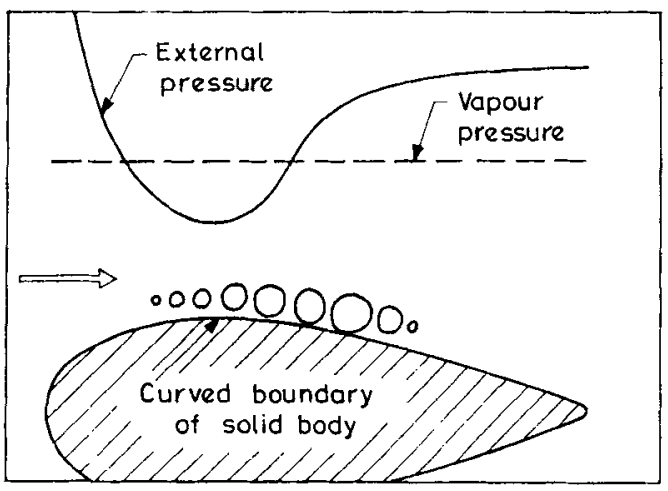

Figure 10. Sketch illustrating growth and collapse of a cavitation bubble subject to external pressure field indicated. 


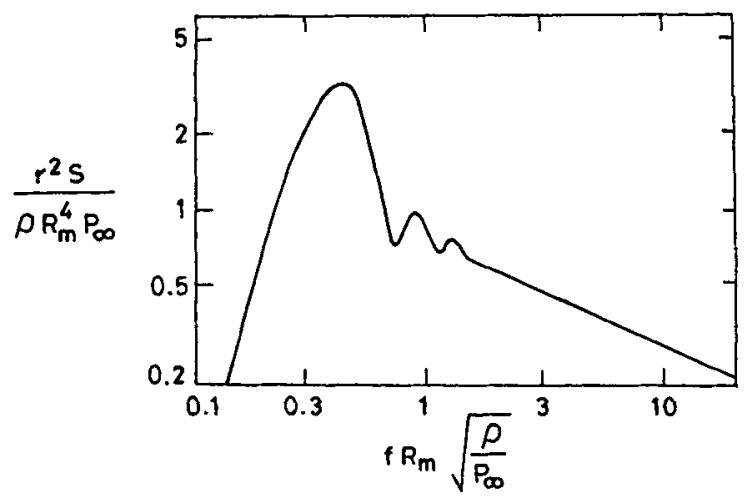

Figure 11. Spectral distribution of single bubble cavitation noise (Fitzpatrick \& Strasberg 1956).

where $\ddot{\mathcal{Q}}$ is the volumetric acceleration of the cavitation zone. Since sound velocity, $c$, in liquids is high, the above to a very good approximation can be written as

$$
P_{a}(r, t)=\frac{\rho_{l}}{4 \pi} \frac{\ddot{\mathcal{Q}}(t)}{r} .
$$

The corresponding spectral energy density for single bubble cavitation noise is given by

$$
S_{b}=\left[\frac{\rho_{l}}{4 \pi r}\right]^{2} \omega^{4}\left|\int_{-\infty}^{\infty} Q(t) \exp [-j \omega t] \mathrm{d} t\right|^{2}
$$

where $\omega=2 \pi f$ is the circular frequency. In certain situations, the volume of cavitation bubble as a function of time, $Q(t)$, can be computed from the solution of the bubble dynamics equation and one of the first such solutions is due to Plesset (1949). Using a typical volume dependence on time of the type computed by Plesset (1949), Fitzpatrick Strasberg (1956) obtained the spectrum for single bubble cavitation noise which is reproduced in figure 11 . Some of the important features of single bubble cavitation noise are:

(1) The spectral density at low frequencies $\left(f<f_{\max }\right)$ increases as $f^{4}$.

(2) The spectral density has a peak at about $f=f_{\max } \sim 0.5 / \tau_{c}$, where $\tau_{c}$ is a time scale proportional to the bubble collapse time.

(3) The spectral density decays as $f^{-0.4}$ for $f>f_{\max }$.

The high frequency dependence noted above is only valid up to a certain frequency since it is based on the assumption of incompressibility of the liquid medium. However, during the final stages of collapse, compressible effects can become important which results in the modification of the spectrum and the decay of the spectral density is more like $f^{-2}$ at the highest frequencies. Further, from the work of Fitzpatrick \& Strasberg (1956) we note that the parameters of relevance in normalizing the spectrum and the frequency are the maximum bubble radius, $R_{m}$, and the collapse time, $\tau_{c}$, respectively.

In reality, cavitation noise consists of several events and it is common practice in measurements to obtain the resulting mean-square sound pressure, $\overline{P_{s}^{2}}(f, \Delta f)$ at a mean frequency, $f$, and bandwidth, $\Delta f$, with a suitable transducer and instrumentation system. Following Blake et al (1977), a measured sound pressure in a narrow frequency band, $\Delta f$ 
can be interpreted as an energy spectrum,

$$
S(f)=\tau_{l} \frac{\overline{P_{s}^{2}}(f, \Delta f)}{\Delta f},
$$

where $\tau_{l}$ is the bubble life time given by $\gamma \tau_{c}$, with $\gamma$ having a value of about 3 . Thus, the measured cavitation noise spectrum can be presented in a normalized form, similar to that for the single bubble cavitation noise spectrum as:

$$
S\left(f \tau_{c}\right)=\frac{\overline{P_{s}^{2}}(f, \Delta f)}{\Delta f}\left[\frac{\gamma \tau_{c} r^{2}}{R_{m}^{4} \rho_{l} P_{\infty}}\right] .
$$

This in fact has been done by Blake et al (1977) and more recently by Arakeri \& Shanmuganathan (1985), who have shown that the above form is valid only when the interference between neighbouring bubbles is not significant.

On the basis of the normalized form of the cavitation noise spectrum, we can arrive at certain useful conclusions about its parametric dependence and scaling. In general, where cavitation is dominated by bubble growth and collapse events, we can write from (45),

$$
\overline{P_{s}^{2}}(f, \Delta f)=\Psi\left(f \tau_{c}\right) \Delta f\left[\frac{R_{m}^{4} \rho_{l} P_{\infty}}{\gamma \tau_{c} r^{2}}\right] .
$$

Here, for convenience $S\left(f \tau_{c}\right)$ is replaced by a more general function $\Psi\left(f \tau_{c}\right)$. From solutions to the bubble dynamics equation (see, for example, lyer 1985), it can be shown that

$$
\begin{aligned}
R_{m} / L & =f\left(K,\left|C p_{m}\right|\right), \quad \text { and } \\
\tau_{c} U_{\infty} / L & =g\left(K,\left|C p_{m}\right|\right),
\end{aligned}
$$

where $L$ is a characteristic length and $f$ and $g$ are arbitrary functions dependent on the parameters $K$, the cavitation number and $\left|C p_{m}\right|$, the absolute value of the minimum pressure coefficient. Substituting the above in (46), we obtain for $\overline{P_{s}^{2}}(f, \Delta f)$,

$$
\overline{P_{s}^{2}}(f, \Delta f)=\Psi\left(f \tau_{c}\right) \frac{\Delta f P_{\infty} \rho_{l}}{r^{2}}\left[\frac{L^{4} f^{4}\left(K,\left|C p_{m}\right|\right) U_{\infty}}{\gamma L g\left(K,\left|C p_{m}\right|\right)}\right]
$$

and which can be rewritten in the form

$$
\overline{P_{s}^{2}}(f, \Delta f)=\left(\Delta f / r^{2}\right) P_{\infty} \rho_{l} L^{3} U_{\infty} \Psi_{l}\left(f \tau_{c}, K,\left|C p_{m}\right|\right) .
$$

Now, from the definition of $K$ and ignoring $P_{v}$ as compared to $P_{\infty}$ (for example, $P_{v}$ in the case of water at $20^{\circ} \mathrm{C}$ is of the order of 0.03 bar) we note

$$
P_{\infty}=K\left[\frac{1}{2} \rho_{l} U_{\infty}^{2}\right]
$$

and

$$
U_{\infty}=\sqrt{\frac{2}{K}} \sqrt{\frac{P_{\infty}}{\rho_{l}}}
$$


Substituting for $P_{\infty}$ or $U_{\infty}$ in (49) and absorbing the added dependence on $K$ in $\Psi_{1}$, we get the following two alternate expressions for $\overline{P_{s}^{2}}(f, \Delta f)$,

$$
\overline{P_{s}^{2}}(f, \Delta f)=\left(\Delta f / r^{2}\right) \rho_{l}^{2} U_{\cdot \mathrm{x}}^{3} L^{3} \Psi_{l}\left(f \tau_{c}, K,\left|C p_{m}\right|\right)
$$

and

$$
\overline{P_{s}^{2}}(f, \Delta f)=\left(\Delta f / r^{2}\right) P_{\infty}^{3 / 2} \rho_{l}^{1 / 2} L^{3} \Psi_{l}\left(f \tau_{c}, K,\left|C p_{m}\right|\right) .
$$

In terms of sound pressure levels, $L_{P}$ in $\mathrm{dB}$, denoting the quantities in prototype situation (or at some other conditions) with primes and those from model tests (or at reference conditions) without primes the following scaling laws are possible,

$$
L_{P}^{\prime}=L_{P}+10 \log \frac{\Delta f^{\prime}}{\Delta f}-20 \log \frac{r^{\prime}}{r}+30 \log \frac{U_{\infty}^{\prime}}{U_{\mathrm{x}}}+20 \log \frac{\rho_{l}^{\prime}}{\rho_{l}}+30 \log \frac{L^{\prime}}{L}
$$

and

$$
L_{P}^{\prime}=L_{P}+10 \log \frac{\Delta f^{\prime}}{\Delta f}-20 \log \frac{r^{\prime}}{r}+15 \log \frac{P_{\propto}^{\prime}}{P_{\infty}}+5 \log \frac{\rho_{l}^{\prime}}{\rho_{l}}+30 \log \frac{L^{\prime}}{L},
$$

provided the similarity conditions

$$
\begin{aligned}
K^{\prime} & =K \\
C p_{m}^{\prime} & =C p_{m}, \\
\Psi_{1}^{\prime} & =\Psi_{1}, \\
f & =f \frac{L}{L^{\prime}} \frac{U_{\infty}}{U_{\infty}^{\prime}} \quad \text { or } f \frac{L}{L^{\prime}}\left(\frac{P_{\propto} \rho_{l}^{\prime}}{P_{\propto}^{\prime} \rho_{l}}\right)^{1 / 2},
\end{aligned}
$$

are maintained. The above clearly indicate the parametric dependence of sound pressure levels on different variables. For example, in the use of turbomachines $L$ can be taken as the impeller diameter, $U_{\infty}$; the tip velocity, $P_{\infty}$, the static pressure at inlet and $K$ evaluated from (4).

\subsection{Universal characteristics of cavitation noise}

In the previous section we have arrived at certain useful relationships for scaling of cavitation noise on the basis of consideration of normalized form of single bubble cavitation noise spectrum. These relationships do not allow the prediction of cavitation noise levels, which we consider in this section. This is primarily based on the development due to Blake (1987) and existing data on propeller cavitation noise, which show certain universal characteristics. The general predictions are then possible on specification of only two parameters, which are cavitation type dependent.

The starting point for us here is the expression for $\overline{P_{s}^{2}}(f, \Delta f)$ in the form

$$
\overline{P_{s}^{2}}(f, \Delta f)=G\left(f \tau_{c}, \frac{K}{K_{i}}, K_{i}\right) \Delta f\left[\frac{L_{c}^{4} \rho_{l} P_{x}}{\gamma \tau_{c} r^{2}}\right]
$$

which is a generalized form of (48) with $R_{m}$ replaced by cavity dimension $L_{c}$ and $\Psi\left(f \tau_{c}\right)$ replaced by $G\left(f \tau_{c}, K / K_{l}, K_{i}\right)$. The latter allows for more definite expressions for $L_{c}$ and $\tau_{c}$ 
than the general forms of (47). Blake \& Sevik (1982) suggest, for limited extent of cavitation near inception,

$$
\begin{aligned}
L_{c} & =L\left(K_{i}-K\right)^{1 / 2} \text { and } \\
\tau_{c} & =L_{c}\left(\rho_{l} / P_{\infty}\right)^{1 / 2}=L\left(K_{i}-K\right)^{1 / 2}\left(\rho_{l} / P_{\infty}\right)^{1 / 2} .
\end{aligned}
$$

In most cases of cavitation noise measurements

$$
\Delta f \approx f \text { or } \Delta f \approx f \tau_{c} / \tau_{c} .
$$

Using this and (58), for $B$ uncorrelated propeller or impeller blades we get from (57),

$$
\overline{P_{s}^{2}}(f, \Delta f) \approx\left(B P_{x}^{2} / r\right) L^{2}\left(K_{i}-K\right) G\left(f \tau_{i}, K / K_{i}, K_{i}\right) .
$$

It should be noted that the above is a slightly modified version of (49) since $K_{i} \approx\left|C_{p_{m}}\right|$. The introduction of $K_{i}$ and $\left(K_{i}-K\right)$ explicitly in the above expression is of significance, since this avoids wrong predictions due to scale effects associated with $K_{i}$. It is not difficult to imagine a scenario where at a given $K$, there may be cavitation in the prototype device, whereas, none in the model or vice-versa owing to $\left(K_{i}\right)_{\text {model }}$ not being equal to $\left(K_{i}\right)_{\text {prototype }}$. Thus, scaling of cavitation noise on the basis of $K$ alone, near inception conditions, can lead to difficulties; however, under developed cavitating conditions it may suffice. Blake (1987), suggests that the above expression for $\bar{P}_{s}^{2}(f, \Delta f)$ be written in the following separable form.

$$
\frac{\overline{P_{s}^{2}}(f, \Delta f)}{B P_{\infty}^{2}(L / r)^{2}\left(1-K / K_{i}\right)}=G\left(f L\left(\rho_{l} / P_{x}\right)^{1 / 2}\left(K_{i}-K\right)^{1 / 2}\right) F\left(K_{i}\right) H\left(K / K_{i}\right) .
$$

Based on controlled propeller cavitation noise measurements, he has also determined $F\left(K_{i}\right)=K_{l}^{-3}$ and $H\left(K / K_{i}\right)=\left(K / K_{i}\right)^{4}$ for surface cavitation. Thus, finally the normalized expression for $\overline{P_{s}^{2}}(f, \Delta f)$ is

$$
\frac{\overline{P_{s}^{2}}(f, \Delta f) K_{i}^{3}}{P_{\propto}^{2}(L / r)^{2}\left(1-K / K_{i}\right)\left(K / K_{i}\right)^{4} B}=G\left(f L\left(\rho_{l} / P_{\infty}\right)^{1 / 2}\left(K_{i}-K\right)^{1 / 2}\right) .
$$

Cavitation noise measurements from several propellers by Blake (1987) and more recently by Sharma et al (1990) has shown reasonably good collapse, when plotted in the above form, in particular at higher frequencies, to suggest a universal form described by

$$
\frac{\overline{P_{s}^{2}}(f, \Delta f)}{\overline{P_{m}^{2}}}=G_{1}\left(\frac{f}{f_{m}}\right)
$$

For propellers $L$ is taken to be the propeller diameter $D$ and $K$ is based on a resultant velocity at the location where cavitation first appears. Here, $\overline{P_{m}^{2}}$ is the maximum spectral level at frequency $f_{m}$, that is $\overline{P_{m}^{2}}=\overline{P^{2}}\left(f_{m}, \Delta f\right)$. The shape of the median spectrum given by (62) is shown in figure 12 from the studies of Blake (1987) and Sharma et al (1990). The empirical information needed for predicting the noise spectrum then basically consists of the non-dimensionalized median spectrum shape and values of its maximum level and corresponding

$$
\tilde{P}_{m}=\frac{\overline{P_{m}^{2}} K_{i}^{3}}{P_{x}^{2}} \frac{B^{4}(D / r)^{2}\left(K / K_{i}\right)^{4}\left(1-K / K_{i}\right)}{.}
$$




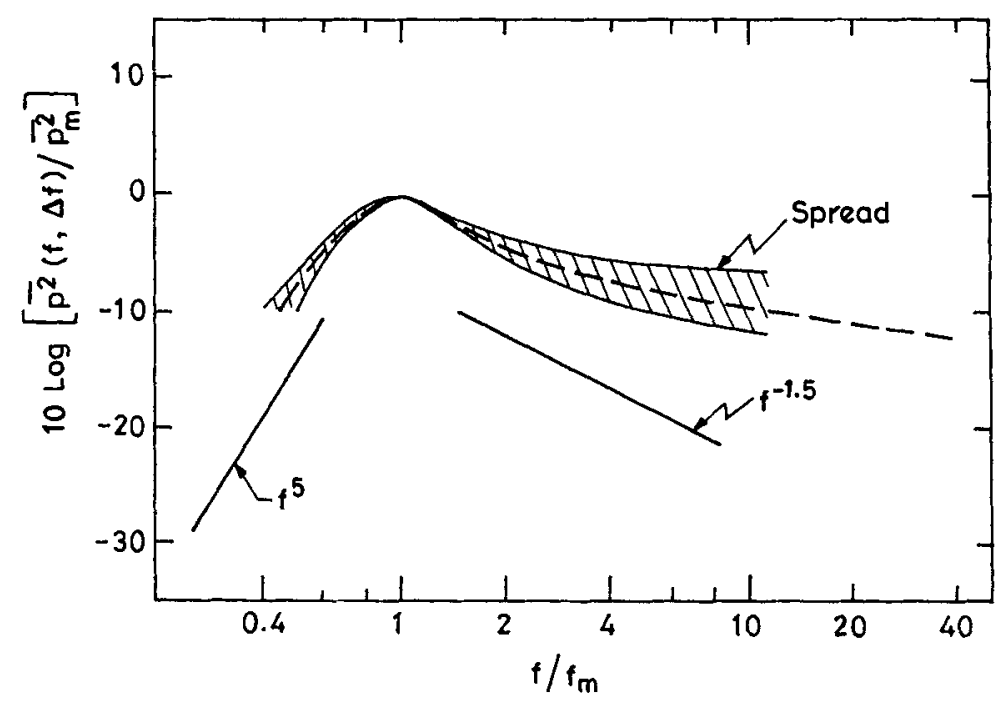

Figure 12. One-third-octave mean spectrum for various types of surface cavitation on marine propellers (Blake 1987 and Sharma et al 1990).

and

$$
\tilde{F}_{m}=f_{m} D\left(\rho_{l} / P_{\infty}\right)^{1 / 2}\left(K_{i}-K\right)^{1 / 2}
$$

The values of $\tilde{P}_{m}$ and $\tilde{F}_{m}$, for different types of cavitation are given in table 5 .

\subsection{Potential use in assessing pump cavitation noise}

The above derived scaling laws and methods of predicting cavitation noise are applicable to free field conditions and the high frequency part of the cavitation noise spectrum. At lower frequencies near blade harmonics, different parameters for scaling are significant. In the case of turbomachines, prediction of cavitation noise must take into account the fact that the levels of interest are those applicable or measured in an enclosure. Therefore, the predicted free field levels, $\bar{P}_{\mathrm{FF}}^{2}(f, \Delta f)$, should be corrected with an appropriate transfer function to obtain the levels, $\overline{P_{e}^{2}}(f, \Delta f)$, within an enclosure. It is expected that the transfer function defined by,

$$
\overline{P_{e}^{2}} / \overline{P_{\mathrm{FF}}^{2}}=\pi\left[f D_{c} / c\right]
$$

Table 5. Values of spectrum parameters $\tilde{P}_{m}$ and $\tilde{F}_{m}$.

\begin{tabular}{lccc}
\hline Cavitation type & $10 \log \tilde{P}_{m}$ & $\tilde{F}_{m}$ & Source \\
\hline Leading edge suction side (LESS) & -95 & 170 & Blake (1987) \\
Leading edge pressure side (LEPS) & -86 & 150 & Blake (1987) \\
Bubble & -67 & 15 & Blake (1987) \\
Leading edge suction side and tip vortex & -78 & 18 & Sharma et al (1990) \\
$\quad$ (LESS or TV) & -52 & 60 & Sharma et al (1990) \\
Cloud (propeller operating in wake) & & & \\
\hline
\end{tabular}


would be a function of non-dimensional frequency as indicated (Blake \& Sevik 1982). For example, in the case of a pump the transfer function could be established with the help of a calibrated source or projector and sensor or receiver combination. First, the projector-receiver combination should be used in a large tank or preferably anechoic facility with the receiver placed at the same distance from the projector as in the actual situation. Then, the same combination with the projector driven at identical voltage(s) as free field conditions should be used in the enclosure with the pump and its piping. The ratio of the two levels then gives the necessary transfer function. For scaling of model results to prototype it may be essential to establish the transfer function for both the model and the prototype. In the case of a pump, the average values based on the projector placed at several potential locations of cavitation should be used. Abbot et al (1987) have recently obtained a transfer function for cavitation noise power levels using the technique mentioned for the MIT water tunnel pump. In addition, they indicate reasonably good agreement between the predicted power levels from an empirical relationship due to Brown (1976) and those inferred from accelerometer and hydrophone measurements along with appropriate transfer functions. The agreement based on accelerometer measurements is better than those based on hydrophone measurements and it is suspected that the presence of air bubbles could be responsible for this finding. Even though it was suggested that the transfer function would be expected to be only dependent on a non-dimensional frequency, it can, in addition, be influenced strongly by the presence of air bubbles, the extent of which is likely to change with physical parameters like cavitation number. Therefore, transfer function determination should be done over an expected range of physical situations. Also, levels corresponding to the expected levels from cavitation noise should be used.

Recent studies of Abbot et al (1987) on water tunnel pump cavitation noise investigations clearly indicate that, with careful choice of transducers and instrumentation system, cavitation onset can be identified, in some cases they have even succeeded in identifying the onset of different types of cavitation in the same pump. The concept of transfer function introduced here is significant and does not seem to have been used by investigators like McNulty \& Pearsall (1979), Kumaraswamy \& Krishna (1987), and others. It should be emphasised that even though the determination of transfer function is a cumbersome procedure, its use enables comparison of cavitation noise measurements from different pumps or turbines.

For illustrative purposes we now use the expression derived earlier along with the results in figure 12 to infer cavitation noise levels in one-third-octave bandwidths for MIT water tunnel pump on which measurements have been reported by Abbot et al (1987). For this purposes, we take $L$ to be the diameter of the impeller and define $K$ as

$$
K=2\left(P_{s}-P_{v}\right) / \rho_{l}\left(N^{2} D^{2} \pi^{2}+V_{A}^{2}\right)
$$

where $P_{s}$ is the static pressure at the impeller centerline and $V_{A}$ is the advance velocity into the impeller. In addition, the following parameters are specified: $D=0.76 \mathrm{~m}, r=D / 2=$ $0.38 \mathrm{~m}, N=314 \mathrm{rpm}(5.23 \mathrm{rps}), P_{\infty}=1.67 \times 10^{5} \mathrm{~N} / \mathrm{m}^{2}, V_{A}=3.49 \mathrm{~m} / \mathrm{s}, B=4, K_{i}=2.63$, and $K=0.58$ (these correspond to $\sigma_{l}$ and $\sigma$ values of 28 and 6.25 respectively in the terminology of Abbot et al (1987)).

Normally, water tunnel pumps operate in the wake of upstream turning vanes, which produce spatially non-uniform velocity fields. In view of this, from table 5 the values 


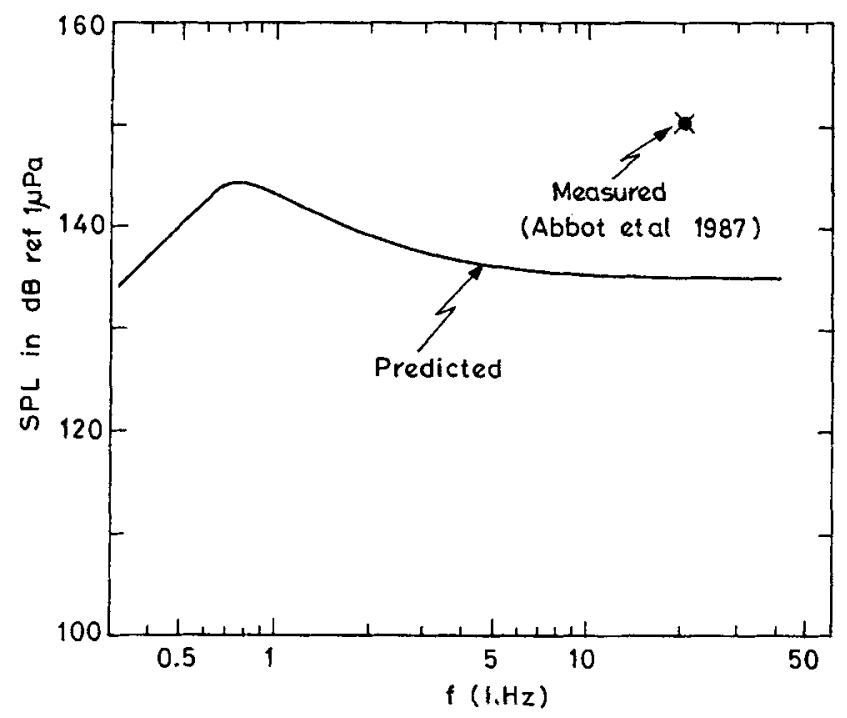

Figure 13. Predicted one-third-octave sound pressure levels for MIT water tunnel pump.

corresponding to propeller operating in the wake are selected; thus

$$
\begin{aligned}
& 10 \log \tilde{P}_{m}=-52 \text { and } \\
& \tilde{F}_{m}=60 .
\end{aligned}
$$

From the definition of the above quantities and using the parameter values noted, we get

$$
\begin{aligned}
& 10 \log \tilde{P}_{m}=-52=10 \log \left[\overline{P_{m}^{2}} \times 2.27 \times 10^{-8}\right] \text { and } \\
& \tilde{F}_{m}=60=f_{m} \times 0.0838 .
\end{aligned}
$$

This allows one to compute the value of $10 \log \overline{P_{m}^{2}}$ and $f_{m}$. Using the results of figure 12 , it is possible to obtain the predicted sound pressure levels versus frequency as shown in figure 13. The measured value from Abbot et al (1987) at $20 \mathrm{kHz}$ and $K=0.58(\sigma=6.25$ in terms of Abbot's definition) is also shown in the same figure. The predicted value being in free field conditions is expected to be lower than measured in an enclosure and, in addition, the exact location of the hydrophone in the experiments is not reported. Considering these, the agreement appears to be quite encouraging.

\subsection{Remarks}

We started with consideration of an idealized situation of single bubble cavitation noise spectrum and ended with very useful information on scaling and prediction of cavitation noise levels from rotating machinery. However, these are in free field conditions and the measurement of transfer function is required to convert them into those in an enclosure. From what has been presented, the parametric dependence of cavitation noise levels in a turbomachine can be predicted provided certain similarity conditions are maintained. The method of presenting cavitation noise data in a suitable normalized and universal form has proved to be highly successful and useful for marine propellers and there is no reason why this should not be the case for turbomachines. 
The article has been prepared with financial support from the Department of Education, Ministry of Human Resource Development through a project on "Marine Propeller Analysis". This support is gratefully acknowledged.

\section{List of symbols}

$A_{s} \quad$ interface cavity surface area;

$B, B_{1} \quad$ thermal cavitation criterion;

$C \quad$ speed of sound;

$C_{A} \quad$ area coefficient;

$C_{i} \quad$ specific heat of liquid;

$C_{0} \quad$ constant;

$C p_{\min }, C p_{m} \quad$ minimum pressure coefficient;

$C_{Q} \quad$ flow coefficient;

$D$ diameter of propeller or impeller;

$D_{c} \quad$ characteristic enclosure dimension;

$f \quad$ frequency or function;

$f_{m} \quad$ frequency at maximum mean spectrum level;

$f_{\max } \quad$ frequency corresponding to maximum level;

F

$g$

gi dimensional constant;

$G, G_{1} \quad$ spectrum functions;

$h$

$h_{s}$

$H$

$H_{a}$

$H_{s}$

$H_{s " \prime}$

$H_{n}$

I

$J$

$k$

$K, K_{i}$

$L$ convective heat transfer coefficient;

height difference between centreline of turbine and level of free surface in the forebay:

head across the machine or function;

atmospheric pressure head;

absolute static pressure head at inlet of pump;

pressure head above vapour pressure head;

vapour pressure head;

sound intensity;

mechanical equivalent of heat;

thermal conductivity

cavitation number in ception caritation number;

$L_{c} \quad$ cavity dimension

$L_{I} \quad$ sound intensity level;

$L_{P} \quad$ sound pressure level;

$L_{w} \quad$ sound power level;

$\underline{m}_{v} \quad$ mass rate of vapourization;

$M_{n} \quad$ molecular weight of vapour;

$n \quad$ rotational speed (rps);

$n(R) \quad$ nuclei number density;

$N \quad$ rotational speed (rpm);

NPSH net positive suction head; 
$\mathrm{Nu} \quad$ Nusselt number;

$P \quad$ root mean square(rms) sound pressure;

$P_{c} \quad$ critical pressure

$P_{s} \quad$ static pressure at inlet of pump;

$P_{v}$ or $P_{v}\left(T_{\infty}\right) \quad$ vapour pressure at bulk temperature;

$\underline{P}_{\infty} \quad$ free stream or collapse pressure;

$\bar{P}^{2}(f, \Delta f) \quad$ measured mean square sound pressure at frequency $f$ and frequency

$\overline{P_{m}^{2}}$

$\mathrm{Pe}$

$\tilde{P}_{m}$

$Q$

$\mathcal{Q}$

$r$

$R$

$R_{2}, R_{1}$

$R_{m}$

$\bar{R}$

$R^{*}$

$S$

$S_{A}$

$S(f)$

$t$

$T$

$T_{c}$

$T_{L}$

$T_{R}$

$T_{t}$

$T^{*}$

$T_{\infty}$

$U$

$U_{\infty}$

$V$

$V_{A}$

$\frac{V}{W}$

$\alpha$

$\delta_{t}$

$\Delta f$

$\Delta H_{v}$

$\Delta T$

$\Delta x$

$\Delta \sigma$

$\lambda$

$\Lambda$

$\omega$

$\pi$

$\Psi, \Psi_{1}$ bandwidth, $\Delta f$;

maximum mean spectrum level;

Peclet number;

non-dimensional maximum mean spectrum level;

pump flow rate or instantaneous bubble volume;

volumetric acceleration;

radial distance;

measured mean nuclei radius;

larger and smaller value of measured nuclei radius;

maximum bubble radius;

universal gas constant;

characteristic nuclei radius;

coefficient of surface tension;

surface area;

energy spectrum of measured sound;

time;

temperature;

critical temperature;

local temperature near cavity surface;

reduced temperature;

triple point temperature;

temperature at which thermodynamic and dynamic parameters are equal;

bulk temperature or reference temperature;

tip velocity, $\pi D n$;

free stream velocity;

average velocity at the inlet of pump;

advance velocity;

volume;

sound power;

thermal diffusivity;

thermal boundary layer thickness;

frequency bandwidth;

change in vapour pressure;

temperature depression;

length of cavity;

change in cavitation number;

latent heat of vapourization;

dynamic parameter;

circular frequency;

transfer function;

spectrum functions; 


$\begin{array}{ll}\rho & \text { density; } \\ \sigma & \text { Thoma cavitation number, } H_{s v} / H ; \\ \sigma_{b}, K_{b} & \text { breakdown cavitation number; } \\ \sigma_{c}, K_{c} & \text { critical cavitation number; } \\ \sigma_{i}, K_{i} & \text { inception cavitation number; } \\ \Sigma & \text { thermodynamic parameter; } \\ \tau & \text { time scale; } \\ \tau_{c} & \text { bubble collapse time; } \\ \tau_{l} & \text { bubble lifetime; }\end{array}$

\section{Subscripts}

$\begin{array}{ll}l & \text { liquid; } \\ v & \text { vapour; } \\ L & \text { local; } \\ R, \text { ref } & \text { reference; } \\ \text { FF } & \text { free field; } \\ e & \text { enclosure. }\end{array}$

Superscripts

overbar

average value;

prototype or other conditions.

\section{References}

Abbot P A, Greeley D S, Brown N A 1987 Water tunnel pump cavitation noise investigations. ASME Int. Symp. on Cavitation Research Facility and Testing Techniques (New York: ASME) pp 99-107

Acosta A, O'Hern T, Katz J 1986 Some recent trends in cavitation research. Int. Symp. on Cavitation, Sendai, pp 1-8

Arakeri V H 1986 Some new approaches in certain aspects of cavitation research. Adv. Aerospace, Fluid Mechanics and Hydraulics (eds) R E A Arndt, M G Stefan, C Farell, S M Peterson (New York: ASCE) pp 77-86

Arakeri V H, Acosta A J 1973 Viscous effects in the inception of cavitation on axisymmetric bodies. J. Fluids Eng. 95: 519-527

Arakeri V H, Berman P 1989 Observations in the estimation of minimum static pressure in flow past a disk. Fourth Asian Cong. of Fluid Mechanics, Hong Kong

Arakeri V H, Shanmuganathan V 1985 On the evidence for the effect of bubble interference on cavitation noise. J. Fluid Mech. 159: 131-150

Arndt R E A 1981 Cavitation in fluid machinery and hydraulic structures. Annu. Rev. Fluid Mech. 15: $273-528$

Billet M L 1986 The importance and measurement of cavitation nuclei. Advances in Aero. Fluid Mechanics and Hydraulics (eds) R E A Arndt, M G Stefan, C Farell, S M Peterson (New York: ASCE) pp 967-988

Blake W K 1986 Mechanics of flow-induced sound and vibration (New York: Academic Press) vol. 1 and 2

Blake W K 1987 Propeller cavitation noise: The problem of scaling and prediction. ASME Int. Symp. on Cavitation Noise, San Francisco (ASME)

Blake W K, Sevik M 1982 Recent developments in cavitation noise research. ASME Int. Svmp. on Cavitation Noise, (ASME) pp 1-10 
Blake W K, Wolpert M J, Geib F E 1977 Cavitation noise and inception as influenced by boundary layer development on a hydrofoil. J. Fluid Mech. 80: 617-640

Bonnin J R 1974 Thermodynamic effects in cavitation. Inst. of Mech. Eng. Conference on Cavitation, Edinburgh, pp 555-562

Brennen C 1969 The dynamic balances of dissolved air and heat in natural cavity flows. J. Fluid Mech. 37: 115-127

Brennen C 1973 The dynamic behaviour and compliance of a stream of cavitating bubbles. J. Fluids Eng. 95: 533-542

Brown N A 1976 Cavitation Noise Problems and Solutions. Int. Symp. on Shipboard Acoustics, Delft Chivers T C 1969-70 Cavitation in centrifugal pumps. Proc. Inst. Mech. Eng. (London) 184: 37-68

Courbiere P. Defaucheux J, Garnaud P 1982 Acoustic methods in sodium flow for characterizing pump cavitation and noise correlations. ASME Int. Symp. on Cavitation Noise (ASME) pp 127-135

Couston M, Eremeef L R, Vinh P 1987 Quasi three-dimensional analysis for solving cavitation problems at hydropower plants. Proc. Eighth Conf. on Fluid Machinery (Budapest: Hung. Acad. Sci.) pp $167-175$

D'Agostino L. Pham T, Green S 1988 Comparison of a cavitation susceptibility meter and holographic observations for nuclei detection in liquids. Cavitation and multiphase flow forum (ASME) pp 20-24

Fitzpatrick H M, Strasberg M 1956 Hydrodynamic sources of sound. Proc. Symp. Naval Hydrodyn., Washington DC. pp 241-280

Holl J W, Billet M L, Weir D S 1975 Thermodynamic effects on developed cavitation. ASME Symp. on Cavity Flows, Minneapolis (ASME) pp 101-109

Hutton S P, Furness R A 1974 Thermodynamic scale effects in cavitation flows and pumps. Inst. of Mech. Engineers Conference on Cavitation. Edinburgh, pp 329-340

Iyer N 1985 An experimental study on scale effects on cavitation noise. $\mathrm{PhD}$ thesis, Indian Institute of Science, Bangalore

Katz J, O'Hern T J 1983 Cavitation in large scale shear flows. Paper No. 83-FE-33, ASME

Knapp R T, Daily J W, Hammit F G 1970 Cavitation (New York: McGraw Hill) chap. 3

Kuiper G 1981 Cavitation inception on ship propeller models. NSMB Publication No. 655, Netherlands

Kuiper G 1985 Reflections on cavitation inception. Cavitation and multiphase flow forum (ASME) pp $1-14$

Kumaraswamy S 1986 Cavitation studies in centrifugal pumps. PhD thesis, Indian Institute of Technology Madras

Kumaraswamy S, Krishna H C R 1987 Techniques of measurement and analysis of noise due to cavitation in centrifugal pumps. ASME Int. Symp. on Cavitation Research Facilities and Testing Techniques (ASME) pp. 131-137

Lecoffre Y, Bonin J 1979 Cavitation tests and nucleation control. Int. Symp. on Cavitation Inception (ASME) pp 141-146

Leggart L J, Sponagle N C 1982 The study of propeller cavitation noise using cross-correlation methods. ASME Int. Symp. on Cavitation Noise (ASME) pp 49-59

Martin C S, Rao P V 1982 Application of signal analysis to cavitation. ASME Int. Symp. on Cavitation Noise (ASME) pp 109-113

McNulty P J, Pearsall I S 1979 Cavitation inception in pumps. ASME Int. Symp. on Cavitation Inception (ASME) pp 163-170

Oldenziel D M 1979 New instrument in cavitation research. ASME Int. Symp. on Cavitation Inception (ASME) pp 111-124

Ooi K K, Acosta A J 1984 The utilization of specially tailored air bubbles as static pressure sensors in a jet. J. Fluids Eng. 106: 459-465

Parkin B R, Kermeen R W 1953 Incipient cavitation and boundary layer interaction on a streamlined body. Cal. Inst. of Tech. Rept. No. E-35.2, Pasadena, CA

Plesset M S 1949 The dynamics of cavitation bubbles. J. Appl. Mech. 16: 277-282 
Plesset M S 1964 Bubble dynamics. Cavitation in real liquids (ed.) R Davies (Amsterdam: Elsevier)

Robertson J M 1969 Cavitation today - An introduction. Proc. of Symp. on Cavitation State of Knowledge (ASME) pp ।-9

Rubin S 1966 Longitudinal instability of liquid rockets due to propulsion feedback (POGO). $J$. Spacecr. Rockets 3: 1186-1195

Ruggeri S R. Moore R D 1969 Method for prediction of pump cavitation performance for various liquids. liquid temperatures and rotative speeds. NASA TN D-5292

Salemann V 1959 Cavitation and NPSH requirements of various fluids. J. Basic. Eng. 81: 167-173

Schiele O, Hergt P, Mollenkopf G 1974 Some views on the different cavitation criteria of a pump. Institution of Mech. Engineers Conf. on Cavitation, Edinburgh, pp 177-184

Sharma S D, Mani K. Arakeri V H 1990 Cavitation noise studies on marine propellers. J. Sound Vibr. 138: $255-283$

Silberman E, Schiebe F R, Mrosla E 1973 The use of standard bodies to measure cavitation strength of water. SAFHL Rept. 141, Univ. of Minnesota. Minneapolis

Spraker W A 1965 The effects of fluid properties on cavitation in centrifugal pumps. J. Eng. Power 87: 309-318

Stepanoff A J 1961 Cavitation in centrifugal pumps with liquids other than water. J. Eng. Power, ASME 83: 79

Stepanoff A J 1965 Cavitation with liquids other than water. Pumps and blowers, Selected advanced topics (New York: John Wiley)

Stepanoff A J, Kawaguchi K 1962 Cavitation properties of liquids. Proc. of IAHR Symp. on Cavitation and Hydraulic Machinery, Sendai, pp 71-85

Van der Meulen J H 1980 Boundary layer and cavitation studies on NACA 16-012 and NACA 4412 hydrofoils. 13th Symp. on Naval Hydraulics. Tokyo

Wislicenus G F 1947 Fluid mechanics of turbomachinery (New York: McGraw-Hill) 